\title{
Gut microbiome changes in overweight male adults following bowel preparation
}

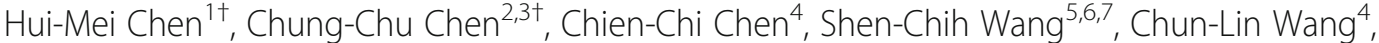 \\ Chien-Hsun Huang ${ }^{4}$, Jong-Shian Liou', Ta-Wei Liư ${ }^{4}$, Hwei-Ling Peng ${ }^{5}$, Feng-Mao Lin ${ }^{1,5}$, Chia-Yuan Liu, 8,9 , \\ Shun-Long Weng ${ }^{9,10,11}$, Chieh-Jen Cheng ${ }^{4}$, Yi-Fang Hung ${ }^{4}$, Chii-Cherng Liao ${ }^{4 *}$ and Hsien-Da Huang ${ }^{12,13,1,5^{*}}$
}

From 29th International Conference on Genome Informatics

Yunnan, China. 3-5 December 2018

\begin{abstract}
Background: Human gut microbiome has an essential role in human health and disease. Although the major dominant microbiota within individuals have been reported, the change of gut microbiome caused by external factors, such as antibiotic use and bowel cleansing, remains unclear. We conducted this study to investigate the change of gut microbiome in overweight male adults after bowel preparation, where none of the participants had been diagnosed with any systemic diseases.

Methods: A total of 20 overweight, male Taiwanese adults were recruited, and all participants were omnivorous. The participants provided fecal samples and blood samples at three time points: prior to bowel preparation, 7 days after colonoscopy, and 28 days after colonoscopy. The microbiota composition in fecal samples was analyzed using $16 \mathrm{~S}$ ribosome RNA gene amplicon sequencing.
\end{abstract}

Results: Our results demonstrated that the relative abundance of the most dominant bacteria hardly changed from prior to bowel preparation to 28 days after colonoscopy. Using the ratio of Prevotella to the sum of Prevotella and Bacteroides in the fecal samples at baseline, the participants were separated into two groups. The fecal samples of the Type 1 group was Bacteroides-dominant, and that of the Type 2 group was Prevotella-dominant with a noticeable presence Bacteroides. Bulleidia appears more in the Type 1 fecal samples, while Akkermensia appears more in the Type 2 fecal samples. Of each type, the gut microbial diversity differed slightly among the three collection times. Additionally, the Type 2 fecal microbiota was temporarily susceptible to bowel cleansing. Predictive functional analysis of microbial community reveals that their activities for the mineral absorption metabolism and arachidonic acid metabolism differed significantly between the two types. Depending on their fecal type, the variance of triglycerides and C-reactive protein also differed between the two types of participants.

Conclusions: Depending upon the fecal type, the microbial diversity and the predictive functional modules of microbial community differed significantly after bowel preparation. In addition, blood biochemical markers presented somewhat associated with fecal type. Therefore, our results might provide some insights as to how knowledge of the microbial community could be used to promote health through personalized clinical treatment.

Keywords: Gut microbiome, Overweight, Bowel preparation, High-throughput sequencing, Bacteroides, Preovotella

\footnotetext{
* Correspondence: Icc@firdi.org.tw; huanghsienda@cuhk.edu.cn

${ }^{\dagger}$ Hui-Mei Chen and Chung-Chu Chen contributed equally to this work.

${ }^{4}$ Food Industry Research and Development Institute, Hsinchu 300, Taiwan

${ }^{12}$ School of Science and Engineering, The Chinese University of Hong Kong,

Guangdong Province, Shenzhen 518172, China

Full list of author information is available at the end of the article
}

(c) The Author(s). 2018 Open Access This article is distributed under the terms of the Creative Commons Attribution 4.0 International License (http://creativecommons.org/licenses/by/4.0/), which permits unrestricted use, distribution, and reproduction in any medium, provided you give appropriate credit to the original author(s) and the source, provide a link to the Creative Commons license, and indicate if changes were made. The Creative Commons Public Domain Dedication waiver (http://creativecommons.org/publicdomain/zero/1.0/) applies to the data made available in this article, unless otherwise stated. 


\section{Background}

Commensal human gut microbiota co-evolve with their host in a symbiotic relationship and influence a large number of biological functions, in particular helping digestion and developing the immune system. Advances in both sequencing technologies and bioinformatics tools have greatly improved our knowledge of the role of gut microbiome in human health and disease [1-3]. In recent years, modulation of gut microbiome has been considered one of the methods of improving health. However, the change of gut microbiome caused by external factors, such as antibiotic use and bowel cleansing, remains unclear. The gut microbial composition is strongly correlated with environmental factors such as dietary habit and drug usage $[4,5]$. The gut microbiome is also linked to host weight $[6,7]$. In Taiwan, over $49.9 \%$ of Taiwanese males are either overweight or obese, with a body mass index (BMI) greater than or equal to $25\left(\mathrm{~kg} / \mathrm{m}^{2}\right)$, as reported by the Health Promotion Administration of Taiwan in 2016. Therefore, further understanding of the composition of gut microbiota and the change of gut microbiome within overweight individuals is needed in order to modulate gut microbiome via external strategies such as probiotics or bowel cleansing.

Due to the high risk of metabolic diseases within people who are either overweight or obese [8], the gut microbiome of the obese is of particular interest. In 2005, Ley and her colleagues reported that obesity was associated with the ratio of the abundance of Bacteroidetes and Firmicutes in genetically obese mice [9]. Subsequently, a decreased proportion of Bacteroidetes and an increased proportion of Frimicutes were observed in obesity [10, 11]. Nevertheless, another study, using fluorescent in situ hybridization, did not support this hypothesis [12]. A large population sample study, called Inter99, revealed that some obese people with lower bacterial richness gained more weight over time [13]; and also found that the population could be separated into two groups differing in the number of their gut microbial genes and the gut bacteria richness. Recently, research in Japanese and Chinese populations also revealed that gut microbial composition differed between obese people and lean people [14-16]. Further, a study using metagenomics sequencing found that obesity-associated gut microbial species is linked to changes in circulating metabolites and that the abundance of Bacteroides thetaiotaomicron decreased in obese people [17].

The effects of probiotic-based treatment are different from person to person [18]. Until now, the most common probiotics include Bifodobacteria, Lactobacilli, Enterococci, and yeasts; and their effect on human health and disease have been reported [19, 20]. For example, Lactobacillus rhamnosus CGMC1.3724 formulation may influence weight loss in obese women [21]; and Lactobacillus gasseri SBT2005 may regulate abdominal adiposity in Japanese populations [22, 23]. In contrast, some Lactobacillus species including $L$. acdophilus might lead to weight gain, not loss [24]. Thus, different species might result in diverse effects. Now fecal material transplantation (FMT) has become another treatment option to modify gut microbiome. Beneficial effects of FMT are shown in patients with Clostridium difficile infections (CDI) or inflammatory bowel disease (IDB) $[25,26]$. In addition, another study showed that insulin sensitivity increases in obese participants with metabolic syndrome after the transfer of intestinal microbiota from lean donors [27]. Additionally, it is noticeable that bowel preparation may change gut microbiome [28-30]. Therefore, it is important to understand the influence of external environmental factors on the variation of gut microbiome.

Inspired by the study of Enterotype and Prevotella-ratios [31, 32], and due to ethnic and cultural variance, we focused on omnivore, overweight, male Taiwanese adults. The two main aims of this study are addressed by the following questions: What are the dominant gut microbiota, and to what extent does microbial diversity change after bowel preparation? Which predictive functional modules in fecal community differ most from before bowel preparation to after colonoscopy? Our results might provide some insights as to how knowledge of the change in microbial community could be used to promote health through personalized clinical treatment.

\section{Methods}

\section{Experimental design and participant selection}

The experimental design is illustrated in Fig. 1. The inclusion criteria of participants were male adults whose age ranged from 20 to 60 years, with body mass index (BMI) equal or greater $25\left(\mathrm{~kg} / \mathrm{m}^{2}\right)$. The exclusion criteria are listed in Additional file 1: Table S1. At screening period, twenty-four overweight male participants were met the entry criteria. During the inspection of colonoscopy, four participants identified as colorectal cancer, or polyps were excluded. Finally, a total of twenty participants completed post-procedure visits for the following analysis.

\section{Bowel preparation}

A low-residue diet that avoids foods containing seeds and other indigestible substances is recommended for three days before the colonoscopy. The split-dose of bowel preparation was administrated by Fleet Phospho-Soda Oral Saline Laxative (sodium phosphate) with water (total 3-4l). The second dose was administered 4 to $5 \mathrm{~h}$ before the planned start of the colonoscopy. All of the patients achieved to the level of good (minimal turbid fluid) or excellent (mucosal detail clearly visible) by Ottawa Bowel Preparation Scale. 


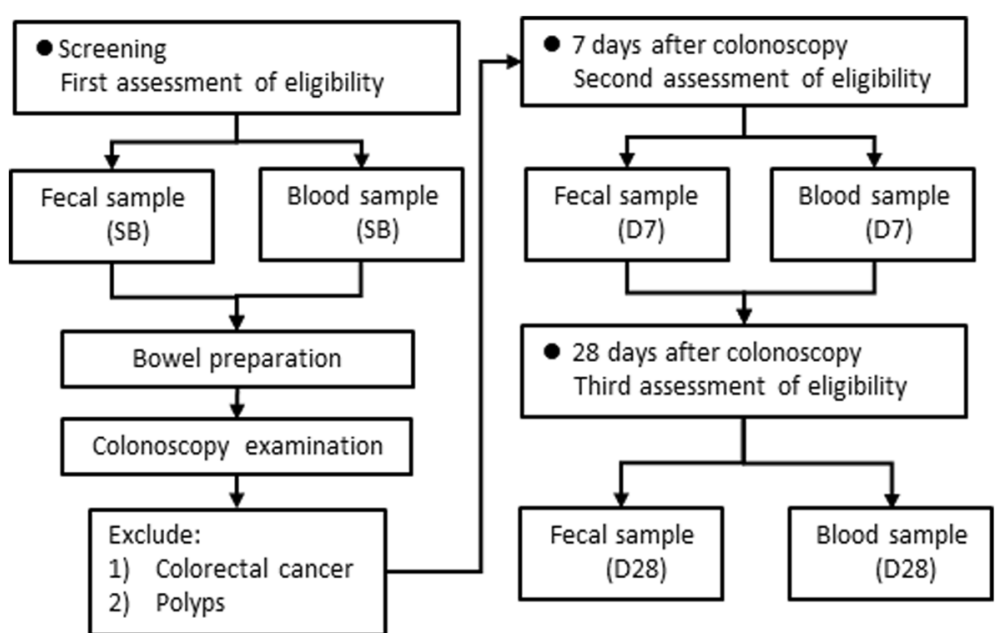

Fig. 1 Experimental design. SB represents baseline, which means the first sample collection time prior to bowel preparation. D7 and D28 represent 7 days and 28 days after colonoscopy, respectively. Samples identified with colorectal cancer or polyps, upon inspection of the colonoscopy, were excluded from the analysis

\section{Fecal samples and blood samples}

Participants provided fecal samples and blood samples prior to bowel preparation, 7 days after colonoscopy, and 28 days after colonoscopy. Fecal samples were collected by participants at home and were put in refrigerator as well as informed a research nurse to deliver these samples to the central laboratory at Food Industry Research and Development Institute. Each fecal sample was collected using two fecal collection devices: one for $5 \mathrm{~g}$ and the other for $15 \mathrm{~g}$. The fecal samples were designed to evaluate the gut microbiota composition as well as fecal calprotectin.

Blood samples were taken after an overnight fast for the determination of plasma lipid profile, fasting plasma glucose (FPG) and immune response. Approximately $14 \mathrm{~mL}$ of blood was collected into three independent blood drawing tubes: $3 \mathrm{~mL}$ for complete blood count $(\mathrm{CBC})$ of safety lab tests, $3 \mathrm{~mL}$ for blood biochemistry of safety lab tests, and $8 \mathrm{~mL}$ for inflammatory cytokine analysis. Serum samples were centrifuged and stored at $80^{\circ} \mathrm{C}$ until analysis. Fasting or 2 -h glucose, serum alanine aminotransferase (ALT), aspartate aminotransferase (AST), triglycerides (TG), total cholesterol (TC), high-density lipoprotein (HDL) cholesterol, and low-density lipoprotein (LDL) cholesterol were measured using an autoanalyser (Beckman Coulter AU5800). Serum IL-6 and TNF- $\alpha$ were measured using a MILLIPLEX MAG Human Adipokine Magnetic Bead Panel (Millipore) according to the manufacturer's instructions.

\section{Bacteria DNA extraction}

Fecal samples were stored at $-80{ }^{\circ} \mathrm{C}$ prior to the DNA extraction. Total DNA was extracted from fecal material using a modified protocol of the QiaAmp DNA Mini Stool Kit (Qiagen, Hilden, Germany). Briefly, each sample was centrifuged at 13,000 rpm for $2 \mathrm{~min}$, and the resulting bacterial pellet was resuspended in $180 \mu \mathrm{l}$ of enzyme solution $(20 \mathrm{mg} / \mathrm{ml}$ lysozyme, $20 \mathrm{mM}$ Tris- $\mathrm{HCl}$ [pH 8.0], $2 \mathrm{mM}$ ethylenediaminetetraacetic acid, and $2 \%$ sodium dodecyl sulfate). Lysates were incubated at $37^{\circ} \mathrm{C}$ for $30 \mathrm{~min}$ prior to the addition of $20 \mu \mathrm{l}$ proteinase $\mathrm{K}$ $(25 \mathrm{mg} / \mathrm{ml})$ and $200 \mu \mathrm{l} \mathrm{Buffer} \mathrm{AL}$. Each suspension was subsequently incubated at $56^{\circ} \mathrm{C}$ for $30 \mathrm{~min}$, and for a further $15 \mathrm{~min}$ at $95^{\circ} \mathrm{C}$. The final DNA was eluted with $30 \mu \mathrm{l}$ of Buffer $\mathrm{AE}$, and stored at $-20^{\circ} \mathrm{C}$ for further analysis.

\section{S rRNA gene amplification}

The PCR primers F515 (5'-GTGCCAGCMGCCG CGGTAA-3') and R806 (5'-GGACTACHVGGGTWTC TAAT-3') were designed to amplify the V4 region of the bacterial 16S ribosomal DNA as described previously [33]. PCR amplification was performed in a $50-\mu \mathrm{l}$ reaction volume containing $25 \mu \mathrm{l} 2 \mathrm{X}$ Taq Master Mix (Thermo Scientific), $0.2 \mu \mathrm{M}$ of forward and reverse primer, and $20 \mathrm{ng}$ DNA template. The reaction process increased the initial temperature to $95^{\circ} \mathrm{C}$ for $5 \mathrm{~min}$, followed by 30 cycles of $95^{\circ} \mathrm{C}$ for $30 \mathrm{~s}, 54^{\circ} \mathrm{C}$ for $1 \mathrm{~min}$, and $72^{\circ} \mathrm{C}$ for $1 \mathrm{~min}$ as well as a final extension of $72^{\circ} \mathrm{C}$ for $5 \mathrm{~min}$. Next, amplified products were checked by $2 \%$ agarose gel electrophoresis and ethidium bromide staining. Amplicons were purified using the AMPure XP beads (Agencourt) and quantified using the Qubit dsDNA HS Assay Kit (Thermo Fisher Scientific), all according to the respective manufacturers' instructions. For V4 library preparation, Illumina adapters were attached to the amplicons using the Illumina TruSeq DNA Sample Preparation v2 Kit. Purified libraries were processed for cluster generation and sequencing using the MiSeq system. 
Taxonomy assignment for bacterial 16S rRNA sequences A pipeline combing PANDAseq [34] and QIIME [1] was used to analyze the raw sequences. The 16S rRNA gene sequences were collected from the National Center for Biotechnology Information Sequence Read Archive in August 2012. Using the default values in QIIME and the taxonomic threshold of $97 \%$ in UCLUST [35], high-quality reads were binned into operational taxonomical units (OTUs).

\section{Statistical analysis}

Taxa of the same type were agglomerated at the phylum, class, order, family, and genus levels. The Type 1 and Type 2 groups were classified by a Prevotella ratio of the sum of Bacteroides and Prebotella within the fecal samples at baseline. The microbial diversity was evaluated from two aspects: alpha diversity and beta diversity. In this study, alpha diversity consisted of richness which means the number of genera, and Shannon diversity index, at the genus level. The variances of richness and Shannon diversity index between the two type groups were evaluated by the nonparametric analysis including the Wilcoxon rank sum test and the Kruskal-wallis test. The strength of correlation was measured using the Spearman's rank correlation coefficient. Beta diversity was evaluated using Phylogeny-based UniFrac analysis [36]. The principal coordinate analysis method was used for visualisation based on data reduction of patterns in an $\mathrm{n}$-dimensional dataset. Six correlation networks of specific genera were built based on the Spearman correlation coefficient. Predictive functional profiling of microbial communities using PICRUSt (phylogenetic investigation of communities by reconstruction of unobserved states) was carried out the 10-base logarithm-transformed data for further analysis [37]. The variances of each predictive functional module and blood biochemical test were evaluated by nonparametric analysis including the Wilcoxon rank sum test and the Kruskal-wallis test. The adjust- $P$ values were evaluated by the Bonferroni correction. R software was used for statistical analysis (The R Project for Statistical Computing, Vienna, Austria).

\section{Results}

\section{Characteristics of the study population}

A total of 20 overweight, male Taiwanese adults were recruited. All participants were omnivorous. Their age ranged from 28 to 53 years, with an average of 40.5 years $(\mathrm{SD}=7.03)$; and their body mass index (BMI) ranged from 25.7 to $34.2\left(\mathrm{~kg} / \mathrm{m}^{2}\right)$, with an average BMI of 28.9 $(\mathrm{SD}=2.6)$. All participants did not have systemic diseases such as anemia, hypertension, diabetes mellitus, chronic kidney disease or abnormal liver function. The clinical data and biochemical data were shown in Table 1. Participants provided fecal samples and blood samples at three time points: prior to bowel prep, 7 days after colonoscopy, and 28 days after colonoscopy. For convenience, the three collection time points were denoted as $\mathrm{SB}, \mathrm{D} 7$, and D28, respectively.

\section{Prevotella-ratio separated into two groups}

We analyzed the microbiota composition in fecal samples using $16 \mathrm{~S}$ rRNA gene amplicon sequencing. The number of genera detected in the three time collections of fecal samples was 131 . Of these, 16 genera were detected in every fecal sample collected, regardless of the collection time point (Fig. 2 and Additional file 2: Table S2). According to the results of an average relative abundance in all fecal samples, the most abundant genera were Bacteroides $(31.7 \%$ of all assigned reads) and Prevotella (23.8\%). The next were Phascolarctobacterium (5.5\%), Faecalibacterium (4.9\%), and Megamonas (4.1\%). Figure 3 depicts fecal microbiota composition at the genus level within individuals. It also reveals that Bacteroides and Prevotella abundance in the SB fecal sample is essentially restored 28 days after colonoscopy.

By evaluating the ratio of Prevotella to the sum of Prevotella and Bacteroides within individuals, the SB fecal samples was separated into two groups. One group had a Prevotella-ratio lower than 0.11, denoted as Type 1; and the other had a Provetella-ratio higher than 0.45 , denoted as Type 2 (Table 2). The participants will be separated into two groups depending on their SB fecal type. Based on the two group, the results of the analysis will be explained from two aspects: first, comparing the differences of fecal microbiome between types 1 and 2, at each of the three collection times; and second, over the three collection times, comparing the differences of fecal microbiome of each type separately.

\section{A degree of microbial diversity change in fecal community between the two groups}

In this study, the richness of microbial diversity means the number of genera detected within each individual. In the SB fecal samples, the interquartile range of richness was wider in the Type 1 group than in the Type 2 group, but the difference in richness was not statistically significant (Additional file 3: Figure S1A). However, in the D28 fecal samples, the interquartile range of richness was wider in the Type 2 group than the Type 1 group, and the richness was significantly different between the two types $(P=0.025$, Additional file 3: Figure S1A). As for the Shannon diversity index, there was no statistical difference between the two types at each of the three collection times, but in the SB fecal samples, the interquartile range of Shannon diversity index was wider in the Type 1 group than in the Type 2 group (Additional file 3: Figure S1B and Additional file 4: Table S3). Furthermore, the correlation coefficient between richness and Shannon diversity index of each type became closer from SB to D28 (Additional File 3: Figure S1C). 
Table 1 Characteristics of 20 male samples and biochemical data (Mean values with their standard deviation)

\begin{tabular}{|c|c|c|c|c|c|c|}
\hline & SB & & D7 & & $\mathrm{D} 28$ & \\
\hline & Mean & SD & Mean & SD & Mean & SD \\
\hline Age (years) & 40.50 & 7.03 & 40.50 & 7.03 & 40.50 & 7.03 \\
\hline BMI $\left(\mathrm{kg} / \mathrm{m}^{2}\right)$ & 28.92 & 2.63 & 28.80 & 2.65 & 29.00 & 2.65 \\
\hline $\mathrm{Hgb}(\mathrm{g} / \mathrm{dl})$ & 14.83 & 1.53 & 14.89 & 1.60 & 14.87 & 1.70 \\
\hline Fasting blood sugar (mg/dl) & 98.15 & 11.86 & 101.20 & 12.66 & 102.15 & 16.10 \\
\hline SGPT (IU/L) & 35.00 & 17.65 & 35.50 & 20.13 & 36.50 & 20.25 \\
\hline SGOT (IU/L) & 24.20 & 8.32 & 24.10 & 9.26 & 25.65 & 10.57 \\
\hline Triglyceride (mg/dl) & 182.75 & 92.24 & 181.65 & 93.75 & 185.65 & 101.70 \\
\hline Total cholesterol (mg/dl) & 212.60 & 35.95 & 217.90 & 39.40 & 217.25 & 37.29 \\
\hline HDL cholesterol (mg/dl) & 42.20 & 8.75 & 41.50 & 8.34 & 41.30 & 7.67 \\
\hline LDL cholesterol (mg/dl) & 133.20 & 30.83 & 139.45 & 35.43 & 139.45 & 38.57 \\
\hline C-reactive protein (mg/L) & 0.34 & 0.46 & 0.23 & 0.22 & 0.24 & 0.20 \\
\hline IL-6 (pg/mL) & 2.99 & 3.13 & 3.83 & 9.02 & 2.91 & 4.07 \\
\hline TNF-alpha (pg/mL) & 2.30 & 7.85 & 2.85 & 6.85 & 2.29 & 7.05 \\
\hline
\end{tabular}

$S D$ standard deviation, $B M I$ body mass index, SB baseline, D7 7 days after colonoscopy, D28 28 days after colonoscopy, Hgb hemoglobin, $A S T$ aspartate aminotransaminase, $A L T$ alanine aminotransaminase, $H D L$ high density lipoprotein, $L D L$ low density lipoprotein, $I L-6$ Interleukin-6, TNF-alpha, tumor necrosis factor alpha

Next, in the Type 1 group, both the richness and Shannon diversity index did not show significant differences between any of the collection time points, but the interquartile range of richness decreased over the three collection times (Fig. 4). Unlike in the Type 1 group, the

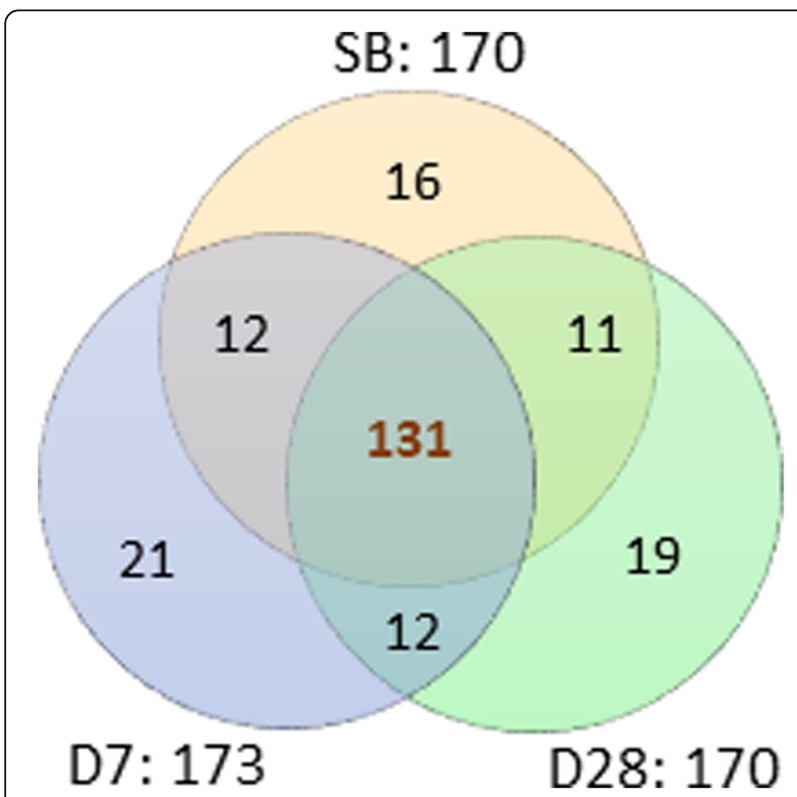

Fig. 2 Venn diagram of the number of genera in the three fecal sample groups. SB, D7, and D28 mean the three sample collection time points: prior to bowel preparation; 7 days after colonoscopy; and 28 days after colonoscopy, respectively. The number of genera detected in all three fecal groups is 131 richness of the Type 2 group significantly differed between SB and D28 $(P<0.032$, Fig. 4A); and the Shannon diversity index significantly differed between $\mathrm{SB}$ and $\mathrm{D} 7(P=0.018)$, but not between SB and D28 (Fig. 4B). In addition, the interquartile range of richness in the Type 2 group increased over the three collection times (Fig. 4B).

According to the results of the weighted UniFrac distance, the 60 fecal samples were separated into two clusters. The Type 1 fecal samples formed one cluster in which the most abundant genus was Bacteroides; and the other group was formed from the Type 2 fecal samples in which the most abundant genus was either Prevotella, Megomonas, or Klebsiella (Fig. 5A and Additional file 5: Fig. S2). Whereas, regardless of the type, the difference of microbial diversity within individuals based on the weighted UniFrac distance did not change remarkably over the three collection times (Fig. 5B).

\section{Significant differences in microbiota between the two groups}

Among the genera which were detected in at least $50 \%$ of the all fecal samples, no genera differed significantly in the Type 1 fecal samples from SB, through D7, to D28. Only four genera: Bacteroides, Prevotella, Oscillospira, Holdemania changed significantly in the Type 2 fecal samples from SB to D7 $(P<0.05)$. In addition, at any sample collection time, Bacteroides and Prevotella differed markedly between the Type 1 and Type 2 groups $(P<0.05)$. The following genera also changed significantly between the Type 1 and Type 2 groups at differing sample collection times: two genera, Akkermansia and Paraprevotella, at SB; five genera, 

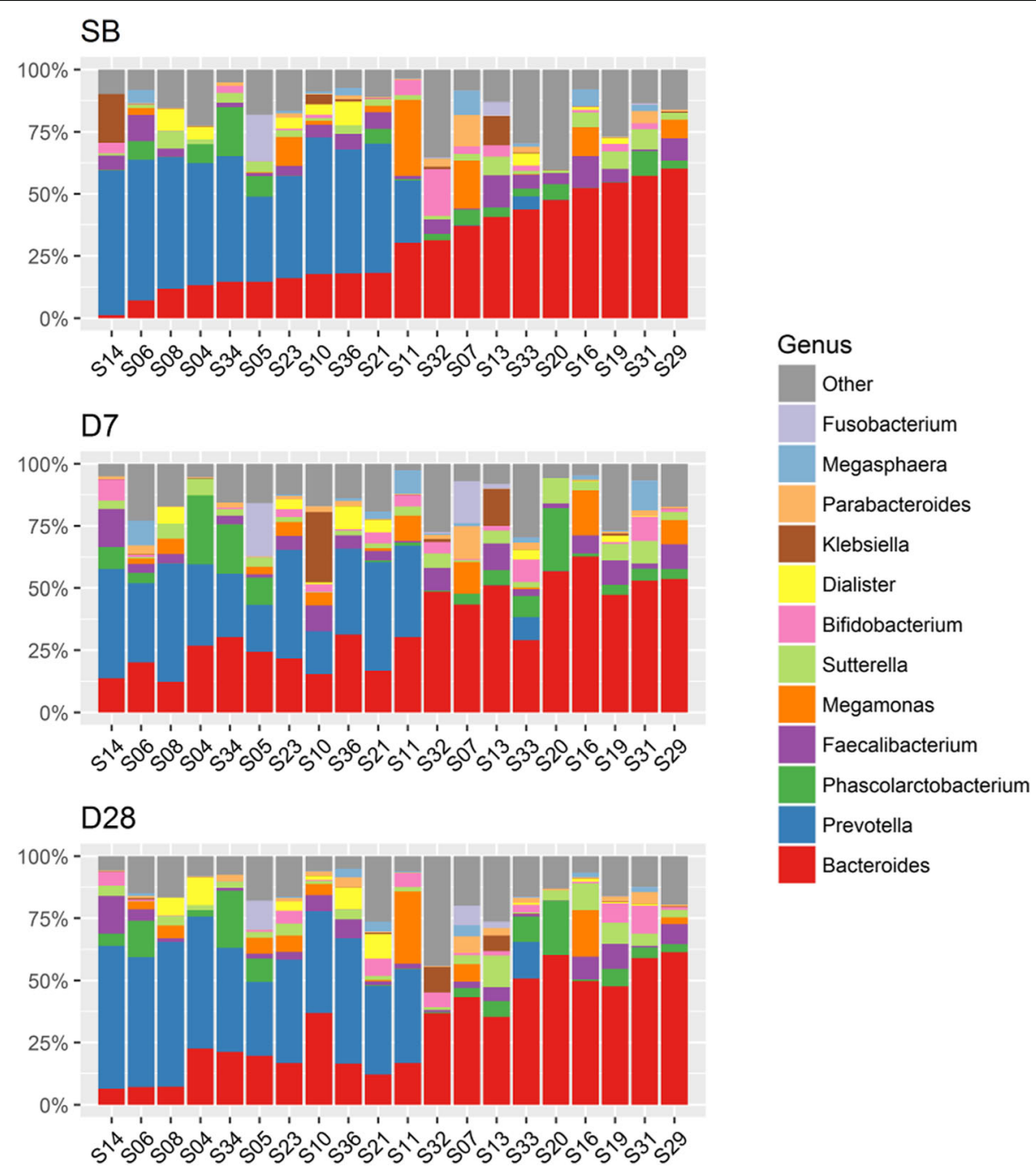

Fig. 3 Microbiota composition of each fecal sample over the three collection times. The fecal microbiota composition profiles at genus level are revealed by 165 rRNA amplicon sequencing. Each color represents one bacterial genus. The two most abundant genera within most individuals were Bacteroides and Prevotella

Akkermansia, Paraprevotella, Actinomyces, Oscillospira, and Fusobacterium at D7; and 3 genera Paraprevotella, Streptococcus, and Enterococcus at D28.

We also found that at the phylum level, the percentage of three phyla, Bacteroidetes, Firmicutes, and Proteobacteria, of each type changed slightly over the three collection times (Fig. 6A). At SB and D7 collection times, the correlations between Bacteroidetes and Firmicutes were negative (Spearman $\rho=-0.53$ and -0.45 , respectively; $P<$ 0.05) (Fig. 6B). At D28, the correlation between Bacteroidetes and Firmicutes was weakly negative without statistical significance (Spearman $\rho=-0.25 ; P=0.27$ ) (Fig. 6B). Furthermore, in the Type 1 group, the correlations were $0.00,-0.40$, and 0.28 at SB, D7, and D28, respectively; and in the Type 2 group, the correlations were $-0.35,-0.58$, and -0.65 at SB, D7, and D28, respectively (Fig. 6C).

\section{A degree of microbial correlation change after bowel preparation}

The correlation networks for 61 genera, which were detected in at least $50 \%$ of the fecal samples at a particular collection time, are presented in the supplementary figures (Additional file 6: Figure S3-S5). The strength of correlation was evaluated using Spearman's correlation coefficient. To further observe the changes of the correlation over the three collection times, the 1830 correlation coefficients were separated into three subintervals $[-1,-0.5],(-0.5,0.5)$, and $[0.5,1]$. An obvious phenomena was that there were fewer pairs of microbiota which remained weakly correlated in the Type 1 group than in the Type 2 group (Additional file 7: Figure S6).

We also found that the correlation of 25 genera, which were detected in at least $90 \%$ of all 60 fecal samples, had 
Table 2 Prevotella ratios of the sum of Bacteroides and Prevotella

\begin{tabular}{lllll}
\hline & & \multicolumn{3}{l}{ Prevotella/(Bacteroides + Prevotella) } \\
\cline { 3 - 5 } ID & Type & SB & D7 & D28 \\
\hline S029 & Type 1 & 0.0001 & 0.0003 & 0.0001 \\
S031 & Type 1 & 0.0001 & 0.0000 & 0.0004 \\
S013 & Type 1 & 0.0004 & 0.0012 & 0.0005 \\
S032 & Type 1 & 0.0005 & 0.0004 & 0.0009 \\
S019 & Type 1 & 0.0006 & 0.0011 & 0.0002 \\
S016 & Type 1 & 0.0006 & 0.0004 & 0.0002 \\
S020 & Type 1 & 0.0027 & 0.0024 & 0.0002 \\
S007 & Type 1 & 0.0033 & 0.0009 & 0.0002 \\
S033 & Type 1 & 0.1066 & 0.2413 & 0.2253 \\
S011 & Type 2 & 0.4544 & 0.5484 & 0.6942 \\
S005 & Type 2 & 0.6990 & 0.4369 & 0.6040 \\
S023 & Type 2 & 0.7182 & 0.6685 & 0.7139 \\
S036 & Type 2 & 0.7343 & 0.5240 & 0.7536 \\
S021 & Type 2 & 0.7405 & 0.7220 & 0.7460 \\
S010 & Type 2 & 0.7554 & 0.5266 & 0.5256 \\
S034 & Type 2 & 0.7752 & 0.4563 & 0.6652 \\
S004 & Type 2 & 0.7872 & 0.5485 & 0.7028 \\
S008 & Type 2 & 0.8165 & 0.7948 & 0.8897 \\
S006 & Type 2 & 0.8880 & 0.6126 & 0.8794 \\
S014 & Type 2 & 0.9802 & 0.7611 & 0.8990 \\
\hline
\end{tabular}

different patterns. From six heat maps of the correlation networks, it is obvious that the pattern of these microbial correlations within the Type 2 group was remarkably different at D7 compared with the other two collection times, whereas these microbial correlations within the Type 1 group seemed not to change significantly (Fig. 7 and Additional file 8: Table S4-S9). It would appear that participants whose fecal type was the Type 2 were temporarily susceptible to bowel preparation.

\section{Variation of predictive functional modules in microbial community between the two types}

Functional potential profiling of microbial communities was evaluated using PICRUSt (phylogenetic investigation of communities by reconstruction of unobserved states) via the KEGG database. At level 3 of the KEGG pathway database, 220 functional modules presented in the SB, D7, and D28 samples. In the SB fecal sample, 26 functional modules were significantly different between the Type 1 group and the Type 2 group $(P<0.05)$, of which mineral absorption and arachidonic acid metabolism differed remarkably $\left(P=1.19 \times 10^{-5}\right.$ and $P=8.34 \times 10^{-5}$, respectively). (Additional file 9: Table $\mathrm{S} 10$ ).

In the Type 1 group, of 220 functional modules, only one module (Bacterial invasion of epithelia cells) changed significantly from SB to D28 (Additional file 10: Table S11). Whereas, in the Type 2 group, 174 functional modules differed significantly between SB and D7 $(P<0.05)$; and 187 between SB and D28 $(P<0.05)$, of which lipopolysaccharide biosynthesis changed remarkably $(P=$ 0.00018, Additional file 11: Table S12). Figure 8 depicts 12 functional modules which differed significantly between the Type 1 and Type 2 groups for each of the three collection times. Using principal component analysis, the 60 fecal samples were mostly separated into two clusters corresponding to the type (Fig. 9A). We also found that the SB and D7 fecal samples were roughly separated into two parts: the first part consisted of $80 \%$ of the SB fecal samples; the second part consisted of $70 \%$ of the D7 fecal samples. As for the D28 fecal samples, $55 \%$ of them were closer to the predominantly SB part and $45 \%$ were in the predominantly D7 part (Fig. 9B).
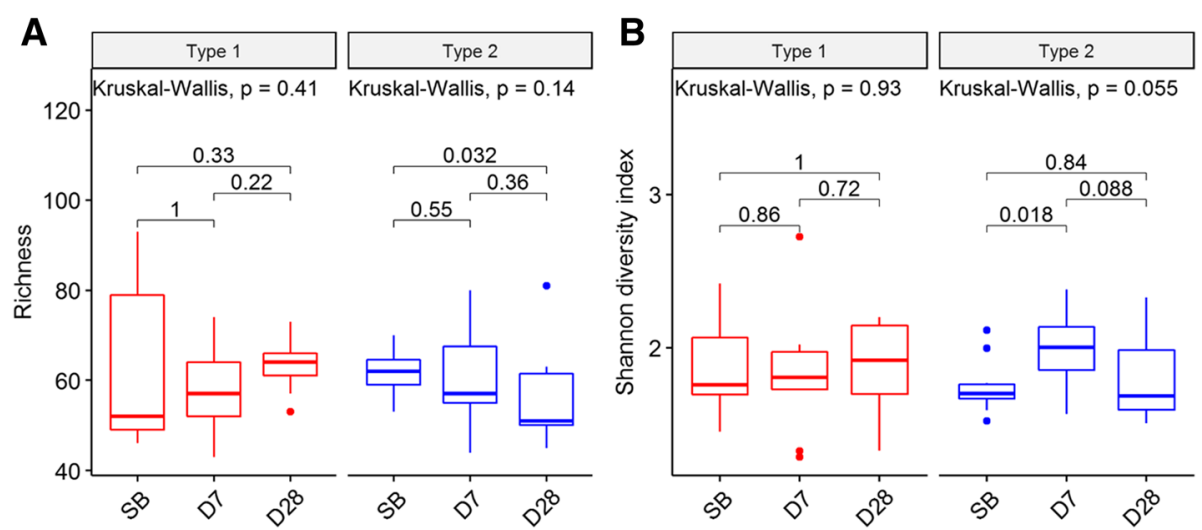

Fig. 4 The change of microbial diversity for each type. (a) Boxplot of richness. In the Type 1 group, the richness does not show significant differences between any of the collection time points, but the spread of richness decreases over the three collection times. In the Type 2 group, the richness significantly differed between SB and D28 $(P=0.032)$. (b) Boxplot of Shannon diversity index. In the Type 1 group, the Shannon diversity index does not show significant differences between any of the collection time points. In the Type 2 group, the Shannon diversity index significantly differs between SB and D7 $(P=0.018)$ 


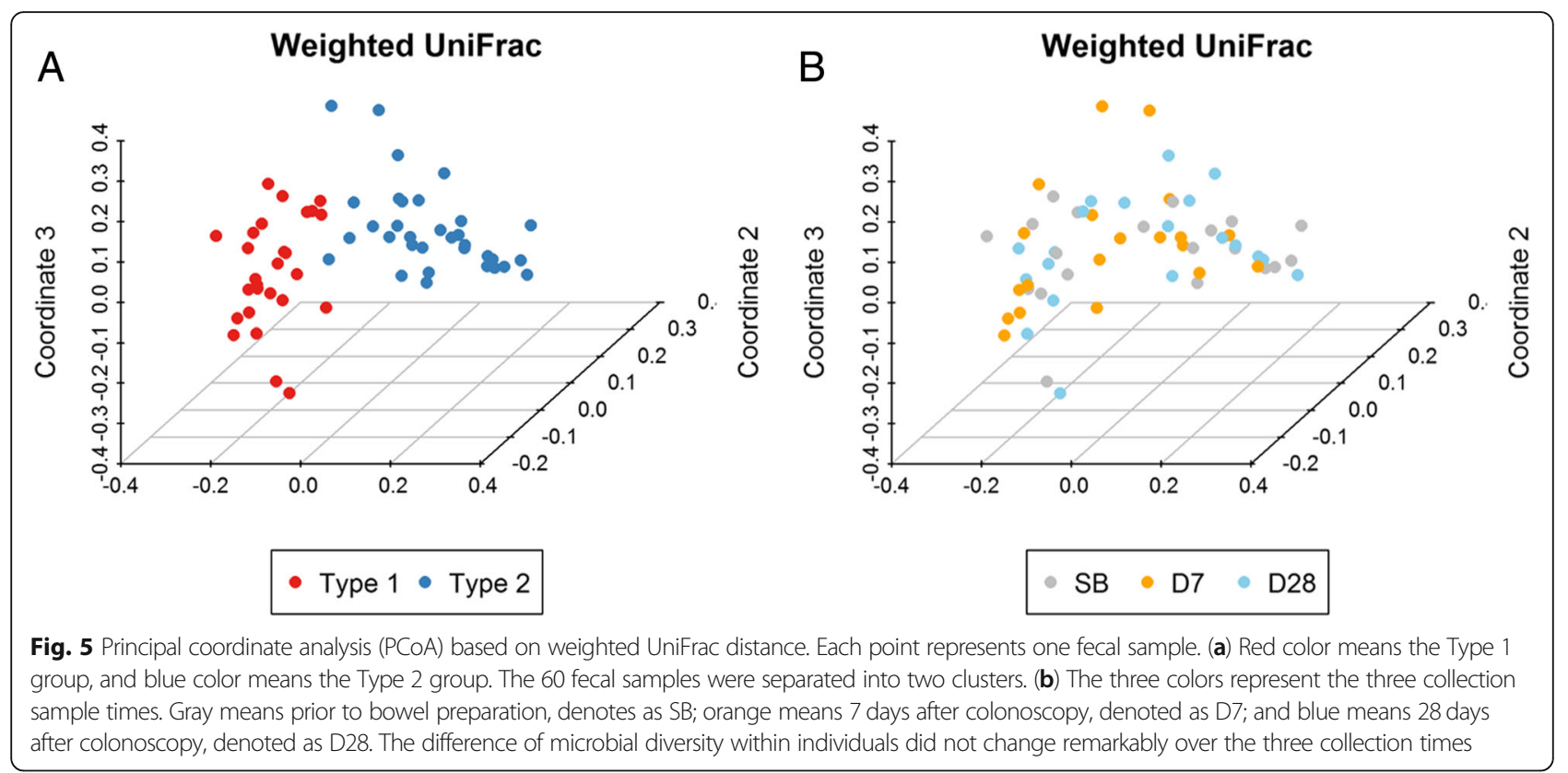

Association of each type with inflammation cytokine and blood tests

In our blood samples, the averages of these inflammation cytokine, including C-reactive protein (CRP), interleukin 6 (IL-6), and tumor necrosis factor alpha (TNF-alpha), were within normal range, but the distribution of the test results differed in the participants of the two types. At all three time points, the participants in the Type 2 group generally had higher median for CRP, IL-6, and TNF-alpha than ones in the Type 1 group (Additional file 12: Figure S7 and

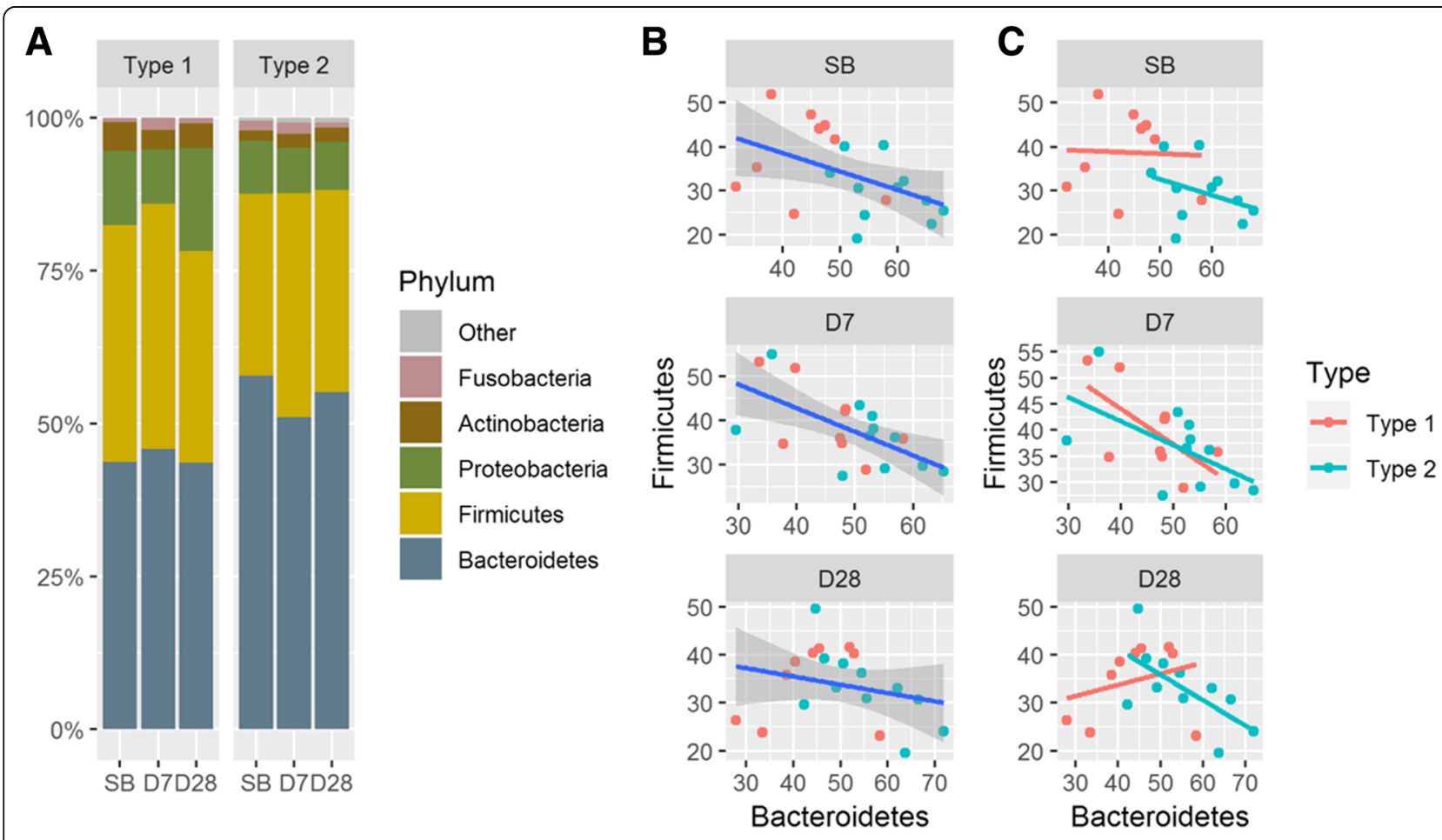

Fig. 6 Microbial distribution at phylum level. (a) Phylum-level microbial composition of fecal community. Two phyla, Bacteroidetes and Firmicutes, are dominant within most individuals. (b) Scatter plots between Bacteroidetes and Firmicutes for each type at the three collection time points. The correlations between Bacteroidetes and Firmicutes at SB and D7 were negative (Spearman $\rho=-0.53$ and -0.45 , respectively; $P<0.05$ ). (c) The correlation between Bacteroidetes and Firmicutes in the Type 1 samples changed markedly from SB to D28 compared with the Type 2 samples 


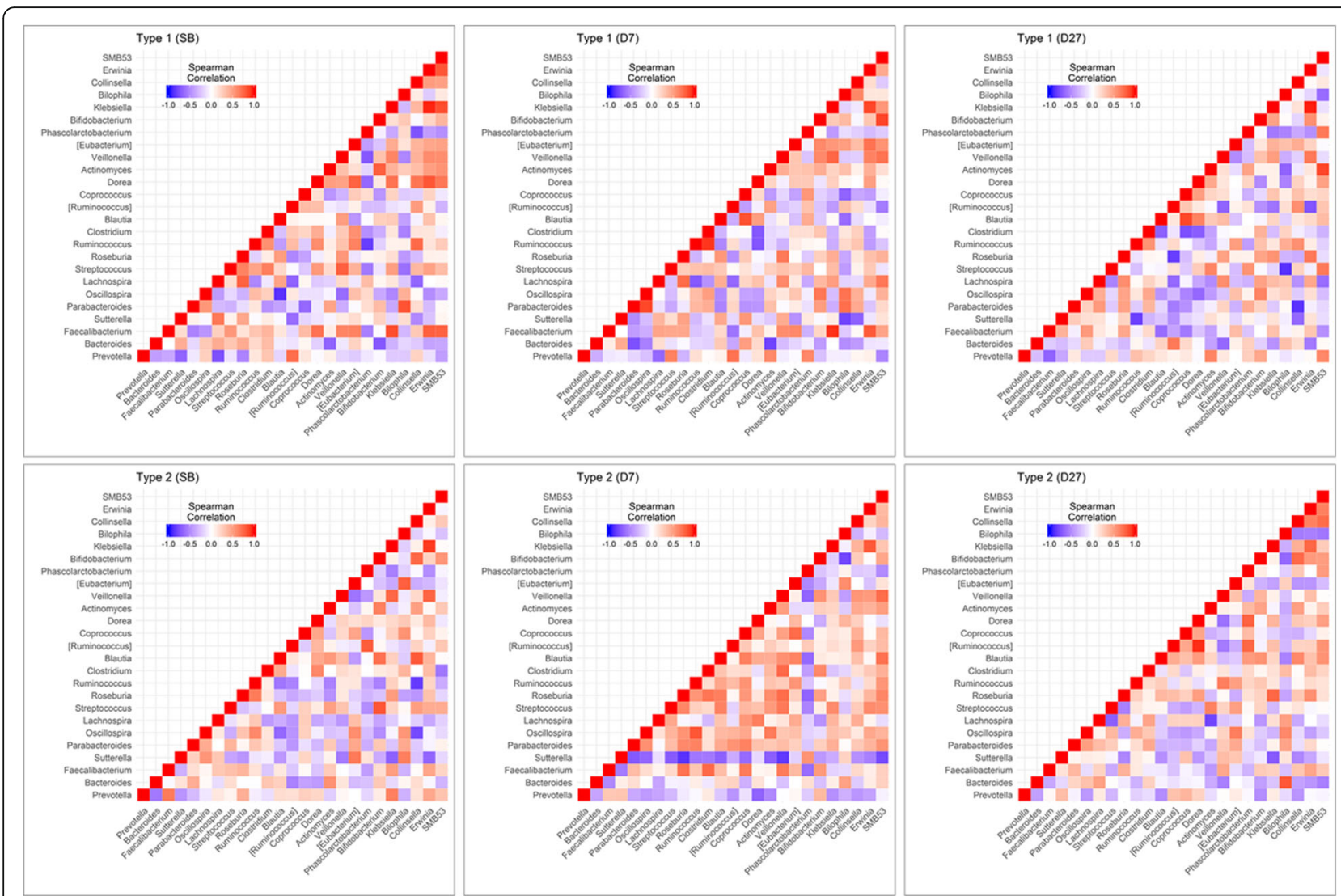

Fig. 7 Six heat maps of correlation networks. Six heat maps of 25 genera which were detected in at least $90 \%$ of all 60 fecal samples. The pattern of theses microbial correlations within the Type 2 group is remarkably different at D7 compared with the other two collection time. Whereas these microbial correlations within the Type 1 group seemed not to change significantly

Additional file 13: Table S13). In addition, the variance of CRP between the Type 1 and Type 2 groups was statistically significant at each sample collection time $(P<0.05$, Additional file 13: Table S13). For each type, by comparing individually CRP, IL-6 and TNF-alpha, there is no statistically significant over the three collection times.

We also found that the variances of liver functions (serum glutamic oxaloacetic transaminase, also known as SGOT; and serum glutamic pyruvic transaminase, also known as SGPT), and the variance of basophils between the two types was significantly different at D7 and at D28 $(P<0.05$, Additional file 14 : Table S14). When comparing each type individually over the three collection times, only the variance of albumin differed significantly in the Type 2 group. Whereas the variance of the other blood tests between the two types generally did not change remarkably (Additional file 14: Table S15). The descriptive statistics of the blood test results present in Additional file 15: Table S16.

Figure 10 depicts the correlations between Bacteroides abundance and each of TG, HDL, and BMI. There were three noteworthy phenomena. First, at each of the sample collection time, Bacteroides negatively correlated with TG in the Type 2 group, whereas Bacteroides positively correlated with TG in the Type 1 group. Furthermore, at SB, Bacteroides positively correlated with HDL in the Type 2 group $(\rho=0.66, P<0.1)$ but very weakly negatively correlated with HDL in the Type 1 group $(\rho=$ - 0.08) without statistical significance. Finally, the correlation between Bacteroides and BMI differed for each type at SB and D7. It was positive in the Type 2 group at $\mathrm{SB}$ and D7. In contrast, it was negative in the Type 1 group at SB but positive at D7.

\section{Discussion}

The purpose of the study was to explore the gut microbiome change in overweight male adults. Our results demonstrated that the most dominant bacteria were hardly influenced by bowel preparation within individuals. However, microbial correlation networks within fecal types were somewhat different over the three sample collection times. In addition, some predictive functional models for microbial community and blood biochemical results within individuals differed between fecal types. 

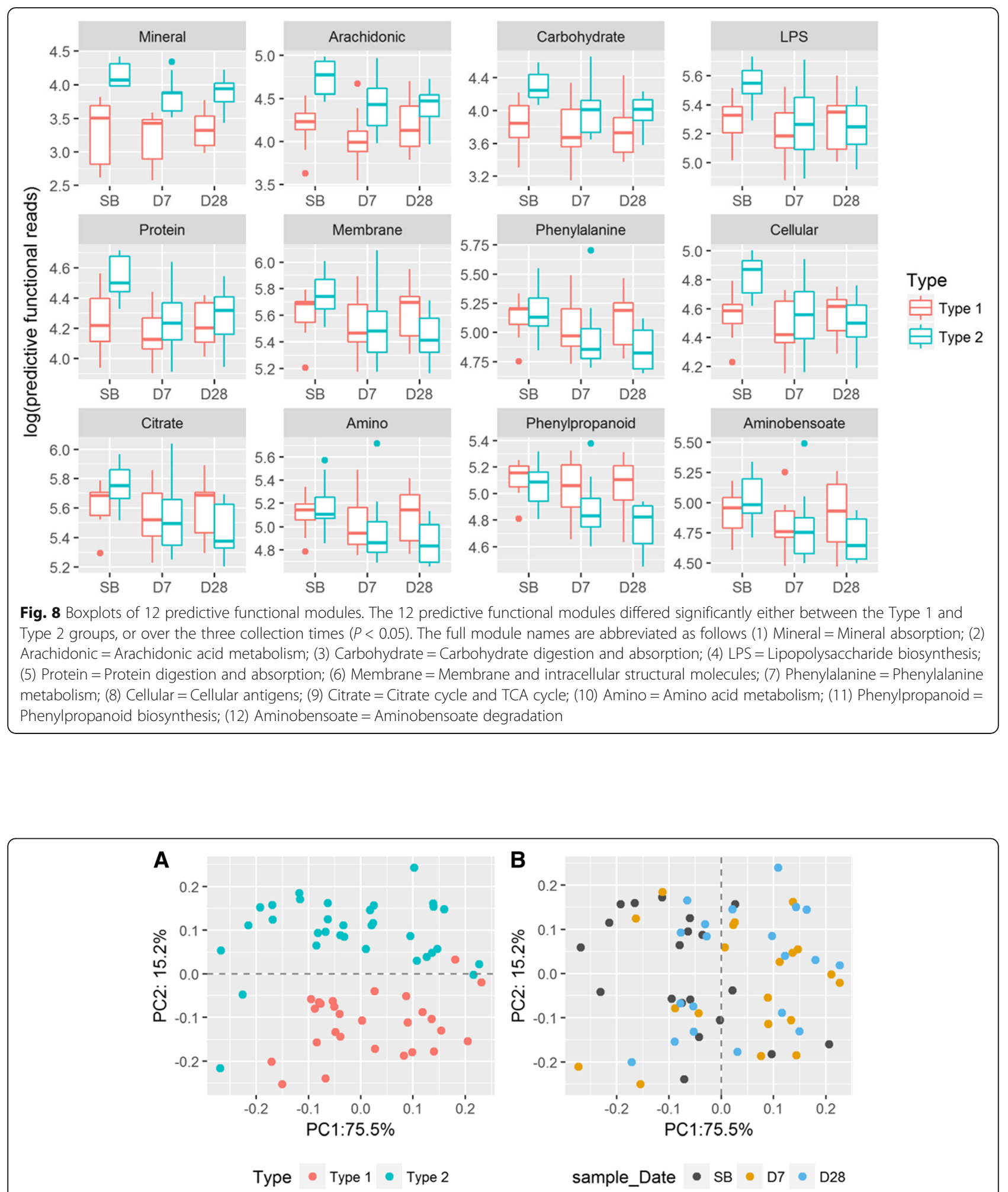

Fig. 9 Principal component analysis of 12 predictive functional modules using PICRUSt in level 3 KEGG database. Each sample is represented by a colored point. (a) Red means type 1 samples, and blue means type 2 samples. The 60 fecal samples are mainly separated into two clusters. (b) Three colors denote the three different sample collection times. Gray means prior to bowel preparation, denoted as SB; orange means 7 days after colonoscopy, denoted as D7; and blue means 28 days after colonoscopy, denoted as D28. The SB and D7 fecal samples are roughly separated into two parts: the first part consists of $80 \%$ of the SB samples; the second part consists of $70 \%$ of the D7 samples. As for the D28 samples, 55\% of them are closer to the predominantly SB part and 45\% are in the predominantly D7 part 

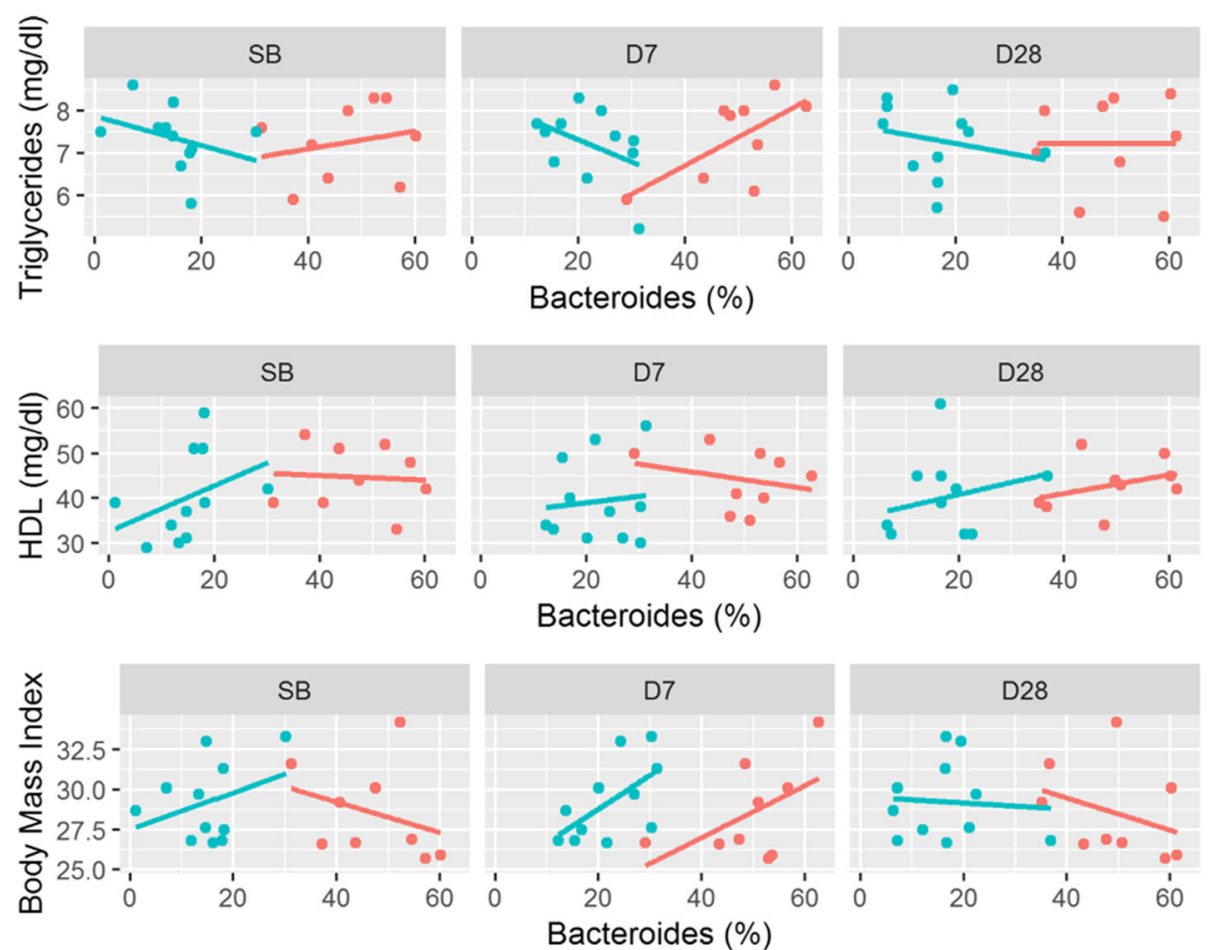

$$
\text { Type } \approx \text { Type } 1 \multimap \text { Type } 2
$$

Fig. 10 Scatter plots between Bacteroides abundance and Triglycerides, HDL, and Body Mass Index. HDL means high-density lipoproteins. Each subject is represented by a colored point. The correlations differed from fecal type at each sample collection time

Clearing the bowel with a controlled diet and a laxative drink before colonoscopy may affect the normal gut microbiome for a short period of time. A study showed that the composition of gut microbiota in 10 middle-aged patients differed significantly after bowel preparation for at least a month [29]. Another case-control study proved that bowel preparation affected the diversity of the fecal and luminal microbiota for weeks [28]. Our results are not fully consistent with the previous findings. In our study, the most dominant bacteria abundances did not change much from prior to bowel preparation to 28 days after colonoscopy. The main reason may be that our participants were all male overweight adults with a healthy colon. In contrast, the subjects of the previous studies did not limit gender and colorectal health status.

Unlike the clustering methods of determining three enterotypes of the human gut microbiome [31, 38], in our study, we used Prevotella relative abundance divided by the sum of Prevotella and Bacteroides $(\mathrm{P} /(\mathrm{P}+\mathrm{B}))$, for to the following reason. Our participants were overweight, and their fecal samples were dominated by either Bacteroides or Prevotella. According to the results of two recent studies, Prevotella and Bacteroides could be biomarkers of diet [39]; and enterotypes could be inferred simply by a Prevotella-to-Bacteroides ratio in persons with central obesity [32]. Thus, we modified a Prevotella-to-Bacteroides $(\mathrm{P} / \mathrm{B})$ ratio into $\mathrm{P} /(\mathrm{P}+\mathrm{B})$ ratio, giving a range from 0 to 1 , which allows us to directly compare the dominance of Prevotella or Bacteriodes.

Bacteroides and Prevotella belong to the phylum Bacteroidetes, which is the most stable component of gastrointestinal microbiota over time in healthy adults [40, 41]. Although our participants were overweight, they were, in general, healthy. Our result is consistent with the previous finding (Fig. 3, Fig. 6A). Another study also showed that the reduced abundance of the Bacteroidetes was linked with obesity $[9,10,42]$. In our study, over the three sample collection times, the correlation between Bacteroidetes and Firmicutes in the Type 1 group changed remarkably while remaining mostly unchanged in the Type 2 (Fig. 6C).

Early research reported that Bacteroides was more common in Western populations [43] and that Prevotella was more common in non-Western populations such as Papua New Guineans [44, 45]. Some researchers further pointed out the potential of reshape the metabolism of Bacteroiedes [46]. In our study, the Type 1 fecal samples were dominated by Bacteroides; and the Type 2 fecal samples were dominated by Prevotella with a noticeable presence of Bacteroides. 
Furthermore, the proportions of Bacteroides-dominant fecal samples and Prevotella-dominant fecal samples were similar. Additionally, the two predictive functional modules, "carbohydrate digestion and absorption" and "mineral absorption", in the Type 2 group had higher levels than in the Type 1 group (Fig. 8). Because our participants were omnivorous and we lacked their diet records, it was a challenge to figure out what causes this phenomenon. However, in Taiwan, the staple diet is white rice [47]. It is rich in carbohydrate and contains minerals such as magnesium, phosphorus, manganese, selenium, iron, folic acid, and thiamine. Further comparing their occupations, we found that the participants in the Type 1 group were all sedentary workers, including programmers, researchers, and project managers. Whereas in the Type 2 group, almost half of the participants were non-office workers, including drivers, manual laborers, and salesmen (Additional file 16: Table S17). Thus, the participants in the Type 2 group might need to ingest more carbohydrate-associated food such as white rice than the participants in the Type 1 group due to the need for more calories for labor.

People who are either overweight or obese are at high risk of metabolic diseases [8]. Akkermansia, Bulleidia, and SMB53 are reported to be associated with obesity. The only current known species within genus Akkermansia is Akkermansia muciniphila, which is a mucin-degrading bacterium [48]. Recent studies indicated that it is associated with body fat mass and glucose intolerance in mice, and that higher $A$. muciniphila seems to be a healthier metabolic status in overweight/obese humans [49]. As for the Bulleidia, it is more abundant in samples with type 2 diabetes (T2DM) and in the early stages of Prediabetes compared to Non-Diabetic samples [50]. In addition, resistant starch or extruded grain result in the enrichment of the relative abundance of Bulleidia [51-53]. The genus SMB53 belongs to the family Clostridiaceae; and a mice model suggests it may be an important factor for the abnormal metabolism of T2DM [54].

In our study, both Akkermansia and Bulleidia differed significantly between the Type 1 and Type 2 fecal samples at SB $(P<0.05)$ but SMB53 did not (Additional file 17: Table S18). Furthermore, the proportions of these genera in each type fecal group were different. At SB fecal samples, Akkermansia appeared in 22\% of the Type 1 and $73 \%$ of the Type 2; Bulleidia appeared in 89\% of the Type 1 and $55 \%$ of the Type 2; and SMB53 appeared in almost all fecal samples. Additionally, the proportions of Akkermansia and SMB53 in each type were similar over the three sample collection times, but that of Bulleidia in the Type 2 group declined remarkably at D7. It seemed that Akkermansia is more likely to appear in the Type 2 fecal samples; and that Bulleidia is more likely to appear in the Type 1 fecal samples. Therefore, it is noted that Akkermansia, Bulleidia, and SMB53 play a role in overweight people who are dominated either by Bacteroides or Prevotella. However, the influence and interaction between these three genera is unclear and requires further investigation.

Recent research showed that some gut bacteria caused substantial variations in triglycerides (TG), high-density lipoproteins (HDL), and BMI [55]. Consistent with this, we also found that Bacteroides, in our study, negatively correlated with TG; positively correlated with HDL; and very weakly correlated with BMI. However, when we separated the participants into two groups based on the Prevotella-ratio, Bacteroides correlated more strongly with each of TG, HDL, and BMI in the Type 2 group than in the Type 1 group at SB and D7(Fig. 10).

Although we focus here on the changes and variations of fecal microbiome in overweight male adults, these findings do not determine the contributions of each genus to the nutrition and health of the host. This study has some limitations. First, due to the number of samples, the findings of this study are restricted to the comparison of two groups corresponding to Prevotella-ratios within individuals. It could be coincident that the range of Prevotella-ratios was either lower than 0.1 or greater than 0.4 . A larger sample size may yield Prevotella-ratios between these two values, or confirm the existence of this split. Second, details of dietary habits and lifestyles were not explored. Our participants were only asked if they were vegetarian or omnivore, and their response was recorded by medical doctors each time participants came back to the hospital during the study period. Further study should include a detailed dietary questionnaire. Then, at least, we could figure out the major differences in meal content between the Type 1 and Type 2 participants. Third, all of our participants were defined as overweight whose BMI were greater than or equal to $25 \mathrm{~kg} / \mathrm{m}^{2}$. However, obese individuals, whose BMI are greater than or equal to $30 \mathrm{~kg} / \mathrm{m}^{2}$, have an excess accumulation of fat, while overweight individuals may or may not. Thus, it might be better to study overweight and obese samples separately. Finally, 16S rRNA gene amplicon sequencing generates the relative abundance of taxa or genes within microbial community. It does not provide absolute abundance information. In addition, biases will occur from the sample processing pipeline [56]. For further study, we should assess the effects of biases for our particular choices of protocols.

\section{Conclusions}

Depending upon the fecal type, the microbial diversity and the predictive functional modules of microbial community differed significantly after bowel preparation. In addition, blood biochemical markers presented somewhat associated with fecal type. Although it is still unclear whether the microbiota composition of feces can accurately reflect the real state of the microbiota composition in the intestine, the gut microbiota by fecal samples might provide clues for health status and might provide to promote health through personalized clinical treatment. 


\section{Additional files}

Additional file 1: Table S1. Exclusion criteria. (PDF $76 \mathrm{~kb}$ )

Additional file 2: Table S2. Percentage of fecal samples and mean relative abundances of the 131 genera. (XLSX $27 \mathrm{~kb}$ )

Additional file 3: Figure S1. The differences of microbial diversity between the type 1 and 2 groups. (PDF $331 \mathrm{~kb}$ )

Additional file 4: Table S3. Descriptive statistics of richness and Shannon diversity index. (PDF $184 \mathrm{~kb}$ )

Additional file 5: Figure S2. Principal coordinate analysis (PCOA) based on the UniFrac distance values. (PDF $414 \mathrm{~kb}$ )

Additional file 6: Figsure S3-S5. Correlation networks for 61 genera at three sample collection times. (PDF $2498 \mathrm{~kb}$ )

Additional file 7: Figure S6. Percentage of the number of correlation change between SB and S7; SB and S28. (PDF $232 \mathrm{~kb}$ )

Additional file 8: Tables S4-S9. Significant correlations for each type group at each sample collection time. (XLSX 27 kb)

Additional file 9: Table S10. Means of 26 predictive functional modules between the Type 1 and Type 2 fecal samples. (PDF 209 kb)

Additional file 10: Table S11. The $P$-values of the predictive functional modules of the Type 1 fecal microbial community. (XLSX 23 kb)

Additional file 11: Table S12. The P-values of the predictive functional modules of the Type 2 Fecal community. (XLSX 23 kb)

Additional file 12: Figure S7. Box plots of three inflammation cytokines. (PDF $377 \mathrm{~kb}$ )

Additional file 13: Table S13. Descriptive statistics of blood test. (XLSX $13 \mathrm{~kb}$ )

Additional file 14: Tables S14 and S15. Table S14: P-values between the Type 1 and Type 2 groups; Table S15: P-values between between any two collection times for each type Table. (PDF $430 \mathrm{~kb}$ )

Additional file 15: Table S16. Descriptive statistics of blood test. (XLSX $17 \mathrm{~kb})$

Additional file 16: Table S17. Participant's lifestyle. (PDF 89 kb)

Additional file 17: Table S18. Percentage of fecal samples and mean relative abundance of SMB53, Bulleidia, and Akkermansia. (PDF $176 \mathrm{~kb}$ )

\section{Abbreviations}

16S rRNA: $16 \mathrm{~S}$ ribosomal RNA; ALT: Serum alanine aminotransferase; AST: Aspartate aminotransferase; BMI: Body mass index; CDI: Clostridium difficile infections; CRP: C-reactive protein; FMT: Fecal material transplantation; HDL: High-density lipoprotein cholesterol; IDB: Inflammatory bowel disease; IL-6: Interleukin 6; OUT: Operational taxonomic units; PICRUSt: Phylogenetic investigation of communities by reconstruction of unobserved states; TG: Triglycerides; TNF-alpha: Tumor necrosis factor alpha

\section{Acknowledgments}

The authors thank the team of Hsinchu MacKay Memorial Hospital (16CT004be), and Food Industry Research and Development Institute (FIRDI_SYN001). We also thank Michael Nicholas for invaluable discussions on data interpretation.

\section{Funding}

The project was funded by the Ministry of Economic Affairs [105-EC-17-A-220643] and by the Hsinchu MacKay Memorial Hospital [MMH-HB-10601, MMHHB-10602]. This work was also supported in part by the Ministry of Health and Welfare [MOHW106-TDU-B-212-144005], by both the Taiwan Ministry of Science and Technology Academic [MOST 106-2319-B-400-001, MOST $107-$ 2321-B-009-001], and by the Novel Bioengineering and Technological Approaches to Solve Two Major Health Problems in Taiwan sponsored by the Taiwan Ministry of Science and Technology Academic Excellent Program (MOST 107-2633-B-009-003). This work was also financially supported by the Center for Intelligent Drug Systems and Smart Bio-devices (IDS2B) from The Featured Areas Research Center Program within the framework of the Higher Education Sprout Project by the Ministry of Education in Taiwan. This article did not receive sponsorship for publication.

\section{Availability of data and materials}

The datasets used and analyzed during the current study are available from the corresponding authors on reasonable request.

\section{About this supplement}

This article has been published as part of BMC Genomics Volume 19 Supplement 10, 2018: Proceedings of the 29th International Conference on Genome Informatics (GIW 2018): genomics. The full contents of the supplement are available online at https://bmcgenomics.biomedcentral.com/ articles/supplements/volume-19-supplement-10.

\section{Authors' contributions}

$\mathrm{CCL}$ served as a principal investigator of the protocol of FIRDI1601. HDH supervised bioinformatics analysis. HMC analyzed the data, preformed data interpretation, and wrote draft. CCC was co-PI and designed the experimental procedure and protocol of FIRD1601. CCC* served as a principal investigator of IRB16CT004b3 and collected clinical samples. HMC and SCW performed the literature research and revised the manuscript. CLW collected clinical data and NGS data. TWL, CHH, JSL, CJC and YFH performed all fecal samples and serum samples for NGS, immune and biochemical assay. HLP performed data interpretation and revised the manuscript. FML performed the sequencing data analysis. CYL collected samples. SLW performed the literature research. CCC*: Chung-Chu Chen. All of the authors have read and approved the final manuscript.

Ethics approval and consent to participate

This study was approved by the Ethical Committee of the Hsinchu MacKay Memorial Hospital (IRB Number: 16CT004be); and the Food Industry Research and Development Institute (Protocol Number: FIRDI1601). All participants gave informed written consent before participating in this study.

\section{Consent for publication}

Not applicable.

Competing interests

The authors declare no competing financial interests.

\section{Publisher's Note}

Springer Nature remains neutral with regard to jurisdictional claims in published maps and institutional affiliations.

\section{Author details}

${ }^{1}$ Institute of Bioinformatics and Systems Biology, National Chiao Tung University, Hsinchu 300, Taiwan. ${ }^{2}$ Division of Hepatology and Gastroenterology, Department of Internal Medicine, MacKay Memorial Hospital, Hsinchu 300, Taiwan. ${ }^{3}$ Teaching Center of Natural Science, Minghsin University of Science and Technology, Hsinchu 300, Taiwan. ${ }^{4}$ Food Industry Research and Development Institute, Hsinchu 300, Taiwan. ${ }^{5}$ Department of Biological Science and Technology, National Chiao Tung University, Hsinchu 300, Taiwan. ${ }^{6}$ Departmnet of Anesthesiology, Taipei Veterans General

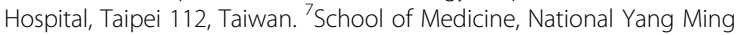
University, Taipei 112, Taiwan. ${ }^{8}$ Division of Gastroenterology, Department of Medicine \& Department of Medical Research, MacKay Memorial Hospital, Taipei 112, Taiwan. ${ }^{9}$ Department of Medicine, MacKay Medical College, New Taipei City 252, Taiwan. ${ }^{10}$ Department of Obstetrics and Gynecology, Hsinchu MacKay Memorial Hospital, Hsinchu 300, Taiwan. ${ }^{11}$ MacKay Medicine, Nursing and Management College, Taipei 112, Taiwan. ${ }^{12}$ School of Science and Engineering, The Chinese University of Hong Kong, Guangdong Province, Shenzhen 518172, China. ${ }^{13}$ Warshel Institute for Computational Biology, The Chinese University of Hong Kong, Guangdong Province, Shenzhen 518172, China.

Published: 31 December 2018

\section{References}

1. Caporaso JG, Kuczynski J, Stombaugh J, Bittinger K, Bushman FD, Costello EK Fierer N, Pena AG, Goodrich JK, Gordon Jl, et al. QIIME allows analysis of highthroughput community sequencing data. Nat Methods. 2010;7(5):335-6.

2. Qin J, Li R, Raes J, Arumugam M, Burgdorf KS, Manichanh C, Nielsen T, Pons $N$, Levenez $F$, Yamada $T$, et al. A human gut microbial gene catalogue established by metagenomic sequencing. Nature. 2010;464(7285):59-65. 
3. Selber-Hnatiw S, Rukundo B, Ahmadi M, Akoubi H, Al-Bizri H, Aliu AF, Ambeaghen TU, Avetisyan L, Bahar I, Baird A, et al. Human gut microbiota: toward an ecology of disease. Front Microbiol. 2017;8:1265.

4. Jernberg C, Lofmark S, Edlund C, Jansson JK. Long-term impacts of antibiotic exposure on the human intestinal microbiota. Microbiology. 2010;156(Pt 11):3216-23.

5. Maier L, Pruteanu M, Kuhn M, Zeller G, Telzerow A, Anderson EE, Brochado $A R$, Fernandez KC, Dose $H$, Mori $H$, et al. Extensive impact of non-antibiotic drugs on human gut bacteria. Nature. 2018.

6. Angelakis E, Armougom F, Million M, Raoult D. The relationship between gut microbiota and weight gain in humans. Future Microbiol. 2012;7(1):91-109.

7. Menni C, Jackson MA, Pallister T, Steves CJ, Spector TD, Valdes AM. Gut microbiome diversity and high-fibre intake are related to lower long-term weight gain. Int J Obes. 2017;41(7):1099-105

8. Weiss R, Dziura J, Burgert TS, Tamborlane W, Taksali SE, Yeckel CW, Allen K, Lopes M, Savoye M, Morrison J, et al. Obesity and the metabolic syndrome in children and adolescents. N Engl J Med. 2004;350(23):2362-74.

9. Ley RE, Backhed F, Turnbaugh P, Lozupone CA, Knight RD, Gordon Jl. Obesity alters gut microbial ecology. Proc Natl Acad Sci U S A. 2005;102(31):11070-5.

10. Ley RE, Turnbaugh PJ, Klein S, Gordon Jl. Microbial ecology: human gut microbes associated with obesity. Nature. 2006;444(7122):1022-3.

11. Verdam FJ, Fuentes S, de Jonge C, Zoetendal EG, Erbil R, Greve JW, Buurman WA, de Vos WM, Rensen SS. Human intestinal microbiota composition is associated with local and systemic inflammation in obesity. Obesity (Silver Spring). 2013;21(12):E607-15.

12. Duncan SH, Lobley GE, Holtrop G, Ince J, Johnstone AM, Louis P, Flint HJ. Human colonic microbiota associated with diet, obesity and weight loss. Int J Obes (Lond). 2008;32(11):1720-4.

13. Le Chatelier E, Nielsen T, Qin J, Prifti E, Hildebrand F, Falony G, Almeida M, Arumugam M, Batto JM, Kennedy S, et al. Richness of human gut microbiome correlates with metabolic markers. Nature. 2013;500(7464):541-6.

14. Kasai C, Sugimoto K, Moritani I, Tanaka J, Oya Y, Inoue H, Tameda M, Shiraki $K$, Ito $M$, Takei $Y$, et al. Comparison of the gut microbiota composition between obese and non-obese individuals in a Japanese population, as analyzed by terminal restriction fragment length polymorphism and nextgeneration sequencing. BMC Gastroenterol. 2015;15:100.

15. Andoh A, Nishida A, Takahashi K, Inatomi O, Imaeda H, Bamba S, Kito K, Sugimoto M, Kobayashi T. Comparison of the gut microbial community between obese and lean peoples using 165 gene sequencing in a Japanese population. J Clin Biochem Nutr. 2016;59(1):65-70.

16. Hou YP, He QQ, Ouyang HM, Peng HS, Wang Q, Li J, LV XF, Zheng YN, Li SC, Liu HL, et al. Human gut microbiota associated with obesity in Chinese children and adolescents. Biomed Res Int. 2017:2017:7585989.

17. Liu R, Hong J, Xu X, Feng Q, Zhang D, Gu Y, Shi J, Zhao S, Liu W, Wang X, et al. Gut microbiome and serum metabolome alterations in obesity and after weight-loss intervention. Nat Med. 2017;23(7):859-68.

18. Gareau MG, Sherman PM, Walker WA. Probiotics and the gut microbiota in intestinal health and disease. Nat Rev Gastroenterol Hepatol. 2010;7(9):503-14.

19. Bron PA, van Baarlen $P$, Kleerebezem M. Emerging molecular insights into the interaction between probiotics and the host intestinal mucosa. Nat Rev Microbiol. 2011;10(1):66-78

20. Varankovich NV, Nickerson MT, Korber DR. Probiotic-based strategies for therapeutic and prophylactic use against multiple gastrointestinal diseases. Front Microbiol. 2015;6:685

21. Sanchez M, Darimont C, Drapeau V, Emady-Azar S, Lepage M, Rezzonico E, Ngom-Bru C, Berger B, Philippe L, Ammon-Zuffrey C, et al. Effect of lactobacillus rhamnosus CGMCC1.3724 supplementation on weight loss and maintenance in obese men and women. Br J Nutr. 2014;111(8):1507-19.

22. Kadooka Y, Sato M, Imaizumi K, Ogawa A, Ikuyama K, Akai Y, Okano M, Kagoshima M, Tsuchida T. Regulation of abdominal adiposity by probiotics (lactobacillus gasseri SBT2055) in adults with obese tendencies in a randomized controlled trial. Eur J Clin Nutr. 2010;64(6):636-43.

23. Ogawa A, Kobayashi T, Sakai F, Kadooka Y, Kawasaki Y. Lactobacillus gasseri SBT2055 suppresses fatty acid release through enlargement of fat emulsion size in vitro and promotes fecal fat excretion in healthy Japanese subjects. Lipids Health Dis. 2015;14:20

24. Million M, Angelakis E, Paul M, Armougom F, Leibovici L, Raoult D. Comparative meta-analysis of the effect of lactobacillus species on weight gain in humans and animals. Microb Pathog. 2012;53(2):100-8.

25. Khoruts A, Sadowsky MJ. Understanding the mechanisms of faecal microbiota transplantation. Nat Rev Gastroenterol Hepatol. 2016;13(9):508-16.
26. Seekatz AM, Aas J, Gessert CE, Rubin TA, Saman DM, Bakken JS, Young VB. Recovery of the gut microbiome following fecal microbiota transplantation. MBio. 2014;5(3):e00893-14

27. Vrieze A, Van Nood E, Holleman F, Salojarvi J, Kootte RS, Bartelsman JF, DallingaThie GM, Ackermans MT, Serlie MJ, Oozeer R, et al. Transfer of intestinal microbiota from lean donors increases insulin sensitivity in individuals with metabolic syndrome. Gastroenterology. 2012;143(4):913-6 e917.

28. Mai V, Greenwald B, Morris JG Jr, Raufman JP, Stine OC. Effect of bowel preparation and colonoscopy on post-procedure intestinal microbiota composition. Gut. 2006;55(12):1822-3.

29. Drago L, Toscano M, De Grandi R, Casini V, Pace F. Persisting changes of intestinal microbiota after bowel lavage and colonoscopy. Eur J Gastroenterol Hepatol. 2016;28(5):532-7.

30. Bachmann R, Leonard D, Delzenne N, Kartheuser A, Cani PD. Novel insight into the role of microbiota in colorectal surgery. Gut. 2017:66(4):738-49.

31. Arumugam $M$, Raes J, Pelletier E, Le Paslier D, Yamada T, Mende DR, Fernandes GR, Tap J, Bruls T, Batto JM, et al. Enterotypes of the human gut microbiome. Nature. 2011;473(7346):174-80

32. Roager HM, Licht TR, Poulsen SK, Larsen TM, Bahl MI. Microbial enterotypes, inferred by the prevotella-to-bacteroides ratio, remained stable during a 6month randomized controlled diet intervention with the new nordic diet Appl Environ Microbiol. 2014;80(3):1142-9.

33. Weng SL, Chiu CM, Lin FM, Huang WC, Liang C, Yang T, Yang TL, Liu CY, Wu WY, Chang YA, et al. Bacterial communities in semen from men of infertile couples: metagenomic sequencing reveals relationships of seminal microbiota to semen quality. PLoS One. 2014;9(10):e110152.

34. Masella AP, Bartram AK, Truszkowski JM, Brown DG, Neufeld JD. PANDAseq: paired-end assembler for illumina sequences. BMC Bioinformatics. 2012;13:31.

35. Edgar RC. Search and clustering orders of magnitude faster than BLAST. Bioinformatics. 2010;26(19):2460-1.

36. Lozupone C, Lladser ME, Knights D, Stombaugh J, Knight R. UniFrac: an effective distance metric for microbial community comparison. ISME J. 2011; 5(2):169-72

37. Langille MG, Zaneveld J, Caporaso JG, McDonald D, Knights D, Reyes JA Clemente JC, Burkepile DE, Vega Thurber RL, Knight R, et al. Predictive functional profiling of microbial communities using $16 \mathrm{~S}$ rRNA marker gene sequences. Nat Biotechnol. 2013;31(9):814-21.

38. Koren O, Knights D, Gonzalez A, Waldron L, Segata N, Knight R, Huttenhower C, Ley RE. A guide to enterotypes across the human body: meta-analysis of microbial community structures in human microbiome datasets. PLoS Comput Biol. 2013;9(1):e1002863.

39. Gorvitovskaia A, Holmes SP, Huse SM. Interpreting Prevotella and Bacteroides as biomarkers of diet and lifestyle. Microbiome. 2016;4:15

40. Lozupone CA, Stombaugh JI, Gordon Jl, Jansson JK, Knight R. Diversity, stability and resilience of the human gut microbiota. Nature. 2012;489(7415):220-30.

41. Rajilic-Stojanovic M, de Vos WM. The first 1000 cultured species of the human gastrointestinal microbiota. FEMS Microbiol Rev. 2014;38(5):9961047

42. Ley RE. Obesity and the human microbiome. Curr Opin Gastroenterol. 2010; 26(1):5-11.

43. Wu GD, Chen J, Hoffmann C, Bittinger K, Chen YY, Keilbaugh SA, Bewtra M, Knights D, Walters WA, Knight R, et al. Linking long-term dietary patterns with gut microbial enterotypes. Science. 2011;334(6052):105-8.

44. Martinez I, Stegen JC, Maldonado-Gomez MX, Eren AM, Siba PM, Greenhill AR, Walter J. The gut microbiota of rural Papua new guineans: composition, diversity patterns, and ecological processes. Cell Rep. 2015;11(4):527-38.

45. Ley RE. Gut microbiota in 2015: Prevotella in the gut: choose carefully. Nat Rev Gastroenterol Hepatol. 2016;13(2):69-70.

46. Rios-Covian D, Salazar N, Gueimonde M, De Los Reyes-Gavilan CG. shaping the metabolism of intestinal Bacteroides population through diet to improve human health. Front Microbiol. 2017:8:376.

47. Pan WH, Wu HJ, Yeh CJ, Chuang SY, Chang HY, Yeh NH, Hsieh YT. Diet and health trends in Taiwan: comparison of two nutrition and health surveys from 1993-1996 and 2005-2008. Asia Pac J Clin Nutr. 2011:20(2):238-50.

48. Belzer C, de Vos WM. Microbes inside--from diversity to function: the case of Akkermansia. ISME J. 2012;6(8):1449-58.

49. Dao MC, Everard A, Aron-Wisnewsky J, Sokolovska N, Prifti E, Verger EO, Kayser BD, Levenez F, Chilloux J, Hoyles L, et al. Akkermansia muciniphila and improved metabolic health during a dietary intervention in obesity: relationship with gut microbiome richness and ecology. Gut. 2016;65(3): 426-36. 
50. Lambeth SM, Carson T, Lowe J, Ramaraj T, Leff JW, Luo L, Bell CJ, Shah VO Composition, Diversity and Abundance of gut microbiome in prediabetes and type 2 diabetes. J Diabetes Obes. 2015;2(3):1-7.

51. Umu OC, Frank JA, Fangel JU, Oostindjer M, da Silva CS, Bolhuis EJ, Bosch G, Willats WG, Pope PB, Diep DB. Resistant starch diet induces change in the swine microbiome and a predominance of beneficial bacterial populations. Microbiome. 2015;3:16.

52. Moen B, Berget I, Rud I, Hole AS, Kjos NP, Sahlstrom S. Extrusion of barley and oat influence the fecal microbiota and SCFA profile of growing pigs. Food Funct. 2016;7(2):1024-32.

53. Umu OCO, Rudi K, Diep DB. Modulation of the gut microbiota by prebiotic fibres and bacteriocins. Microb Ecol Health Dis. 2017;28(1):1348886.

54. Horie M, Miura T, Hirakata S, Hosoyama A, Sugino S, Umeno A, Murotomi K, Yoshida Y, Koike T. Comparative analysis of the intestinal flora in type 2 diabetes and nondiabetic mice. Exp Anim. 2017:66(4):405-16.

55. Fu J, Bonder MJ, Cenit MC, Tigchelaar EF, Maatman A, Dekens JA, Brandsma E, Marczynska J, Imhann F, Weersma RK, et al. The gut microbiome contributes to a substantial proportion of the variation in blood lipids. Circ Res. 2015;117(9):817-24.

56. Brooks JP, Edwards DJ, Harwich MD Jr, Rivera MC, Fettweis JM, Serrano MG, Reris RA, Sheth NU, Huang B, Girerd P, et al. The truth about metagenomics: quantifying and counteracting bias in $16 \mathrm{~S}$ rRNA studies. BMC Microbiol. 2015;15:66.

Ready to submit your research? Choose BMC and benefit from:

- fast, convenient online submission

- thorough peer review by experienced researchers in your field

- rapid publication on acceptance

- support for research data, including large and complex data types

- gold Open Access which fosters wider collaboration and increased citations

- maximum visibility for your research: over $100 \mathrm{M}$ website views per year

At $\mathrm{BMC}$, research is always in progress.

Learn more biomedcentral.com/submissions 